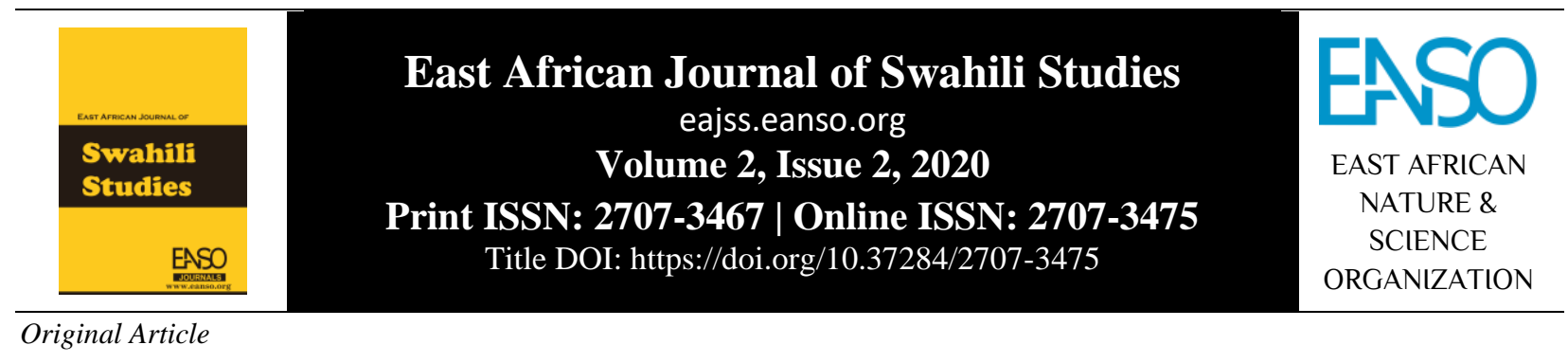

\title{
Maudhui katika Nyimbo za Tohara za Wamasaaba Nchini Uganda
}

\author{
Dr. Willy Wanyenya, $P h D^{1^{*}}$ \\ ${ }^{1}$ Makerere University Business School, P. O. Box 1337, Kampala, Uganda. \\ *Barua pepe ya mawasiliano: willywanyenya@gmail.com \\ ORCID: https://orcid.org/0000-0002-7594-8256
}

DOI ya Nakala: https://doi.org/10.37284/eajss.2.2.194

Tarehe ya Uchapishaji: IKISIRI

13 Agosti 2020 Katika makala hii, mwandishi anaeleza maudhui mbalimbali yanayopatikana katika nyimbo za tohara za Wamasaaba nchini Uganda. Alifanya hivyo kwa

Maneno Muhimu:

Maudhui,

Tohara,

Matukio,

Miti Shamba,

Khukhubuluula. kurejelea angalau mfano mmoja wa nyimbo hizo kwa kila maudhui. Mwandishi amepanga maudhui hayo kulingana na utaratibu wa utendaji wa matukio ya tohara. Kutokana na jambo hili, mwandishi ameangalia matukio mbalimbali katika utekelezaji wa tohara kwa sababu nyimbo hizo huambatana na matukio fulani. Kwa kufanya hivyo, mwandishi ametaja tukio na kutoa mfano wa wimbo unaoimbwa. Baada ya kutoa mfano wa wimbo, anauchanganua na kubainisha maudhui mbalimbali yaliyomo. Mwandishi anafanya hivyo hatua kwa hatua mpaka mwisho. Dhamira ya utafiti ni kueleza jinsi Wamasaaba walivyobobea katika utanzu huu wa sanaa. Hali kadhalika, madhumuni ya utafiti huu ni kueleza namna nyimbo za kiutamaduni zilivyo muhimu katika maisha ya binadamu, kufafanua athari za nyimbo za tohara kisaikolojia na kuonyesha nguvu za mawasiliano za nyimbo za kiutamaduni. Mkabala wa utafiti huu ulikuwa wa nyanjani. Kwa kutumia mkabala huu, mtafiti alizuru nyanjani na kukusanya nyimbo. Katika kazi yake, mtafiti alitumia mbinu ya uchunguzi shiriki. Kwa kutumia mbinu hii, mtafiti alifuatilia makundi ya watendaji na kushuhudia jinsi matukio ya tohara yanavyotekelezwa. Kulingana na aina ya utafiti, mtafiti hakuuliza watu maswali. Mtafiti alitumia kinasa sauti kurekodi nyimbo. Baada ya mchakato huo kukamilika, mtafiti alizichanganua nyimbo ambazo alizikusanya halafu akawasilisha matokeo ya utafiti wake. Nyimbo zilizokusanywa zilijumlisha zile za kupokea wageni, zile zinazoonyesha huzuni kwa mhusika, zile zinazodhihirisha furaha kwa mhusika, zile za kuonya mhusika nk. Mtafiti alianza uchanganuzi wa nyimbo kwa kuziandika kwenye karatasi. Baada ya kufanya hivyo, alizipanga kulingana na madhumuni ya utafiti. Alipomaliza 
kufanya hivyo, mtafiti alieleza maudhui mbalimbali kama yalivyojitokeza katika kila wimbo.

\section{APA CITATION}

Wanyenya, W. (2020). Maudhui katika Nyimbo za Tohara za Wamasaaba Nchini Uganda. East African Journal of Swahili Studies, 2(2), 84-99. https://doi.org/10.37284/eajss.2.2.194

\section{CHICAGO CITATION}

Wanyenya, Willy. 2020. "Maudhui katika Nyimbo za Tohara za Wamasaaba Nchini Uganda". East African Journal of Swahili Studies 2 (2), 84-99. https://doi.org/10.37284/eajss.2.2.194.

\section{HARVARD CITATION}

Wanyenya, W. (2020) "Maudhui katika Nyimbo za Tohara za Wamasaaba Nchini Uganda", East African Journal of Swahili Studies, 2(2), pp. 84-99. doi: 10.37284/eajss.2.2.194.

\section{IEEE CITATION}

W. Wanyenya, "Maudhui katika Nyimbo za Tohara za Wamasaaba Nchini Uganda”, EAJSS, vol. 2, no. 2, pp. 84-99, Aug. 2020.

\section{MLA CITATION}

Wanyenya, Willy. "Maudhui katika Nyimbo za Tohara za Wamasaaba Nchini Uganda”. East African Journal of Swahili Studies, Vol. 2, no. 2, August 2020, pp. 84-99, doi:10.37284/eajss.2.2.194.

\section{UTANGULIZI}

Tunapoongea juu ya nyimbo, mara moja katika mawazo ya mtu ambaye si mwanafasihi panatokea wazo la nyimbo za kisasa kama vile nyimbo za kidini au nyimbo zinazotumbuiza watu katika hafla mbalimbali. Na kwa wanafasihi iwe wasomi au wasiosoma panatokea wazo la nyimbo za kiutamaduni. Ni kweli kwamba kiutamaduni, kuna nyimbo tofautitofauti zinazoambatana na matukio tofautitofauti. Kwa mfano kuna nyimbo za matanga, nyimbo za miviga, nyimbo za kisiasa, nyimbo za matambiko, nyimbo bembelezi na nyimbo za tohara nk.

Kwa vile lengo la makala hii ni kueleza kwa undani maudhui katika nyimbo za tohara za Wamasaaba, makala itaangalia vipengele mbalimbali vya kifasihi katika nyimbo husika. Mwandishi anaeleza jinsi nyimbo hizo zinavyopambwa kwa kutumia vipengele vya kifasihi kama vile kuwepo kwa mafumbo, misemo, tashbihi, isitiari, shada, mahadhi, kurudiwarudiwa kwa maneno nk.

\section{SUALA LA UTAFITI}

Katika kazi hii, suala la utafiti ni kuwa nyimbo za tohara ni nyingi sana. Nyimbo hizi huwa zinabuniwa na vijana wanaotarajiwa kutahiriwa katika kila mwaka wa tohara. Hii ni kwa sababu kila kijana anatarajiwa kubuni wimbo wake. Jambo la kushangaza ni kuwa nyimbo hizi zina vipengele kadhaa wa kadhaa vya kifasihi ambavyo havijatafitiwa. Kwa hiyo, katika makala hii mtafiti anatoa maelezo kuhusu maudhui kama kipengele kimojawapo cha kifasihi.

\section{MAPITIO YA MAANDISHI}

Kulingana na aina mbalimbali za Biblia, katika Agano la Kale, Mfalme Saulo alipigiwa muziki kila aliposumbuliwa na pepo mbaya. Kulingana na Mlacha (1995), tangu zamani, jamii nyingi kote duniani zimekuwa zikiimba nyimbo wakati wa kufanya kazi. Hii ni kwa sababu nyimbo huwaunganisha watu na kuwapa moyo wa kufanya kazi kwa pamoja (Mlacha, 1995). Aidha, nyimbo huimbwa watu wanapokunywa pombe kwa ajili ya kujiburudisha. Nyimbo huwa kama kioo cha jamii. Yaani, mtu akitazama utendaji wa nyimbo, huwa anaiona jamii yenyewe. Kama Nketia (1955) anavyosema, nyimbo ni dirisha ambamo mtu huchungulia na kuiona jamii. Kwa mujibu wa Shilimi (1978), miongoni mwa Wamasaaba, kuna mitazamo tofauti kuhusu nyimbo za tohara. Nyimbo hizi zimesifiwa na baadhi ya watu huku zikikosolewa na wengine. Shilimi (1978) anasema tohara ni tambiko la kuingiza vijana kwenye kundi la wanaume ambapo watu hukusanyika kwa ajili ya kuimba, kucheza, kula na kunywa pombe. Wamara (2000) naye amekosoa nyimbo za tohara akidai kuwa mkusanyiko wa watu husababisha upungufu wa chakula katika jamii. 
Kulingana na Wamara (2000), nyimbo za tohara hutofautiana. Kuna zile za jumla ambazo huimbwa na wafuasi wa wahusika wakuu na zile zinazoimbwa na wahusika wakuu wenyewe. Kulinagana na mtaalamu huyu, Khukhubulula ni zile nyimbo zinazotungwa na kuimbwa na vijana wanaotarajiwa kutahiriwa miongoni mwa Wamasaaba wa Bududa. Nyimbo hizi huimbwa vijana wakiwa njiani wakichezacheza au wakiwa safarini kwenda mahali fulani kwa ajili ya kutembelea jamaa wao.

Kwa mujibu wa Nangoli (2001), kuimba kwa nyimbo hizi ni jambo la kawaida kwa vijana wanaotekeleza tohara kwa njia ya kiutamaduni. Vijana katika Wilaya ya Bududa hubishana katika utunzi wa nyimbo hizo. Hii ni kwa sababu kila kijana huwa na lengo la kuimba na kuwashinda wenzake na hivyo kujulikana katika jamii. Kwa hiyo, nyimbo za tohara hutungwa kwa ujuzi wa hali ya juu sana. Kwa kawaida mhusika mkuu ambaye ni kijana anayetarajiwa kutahiriwa hutangulia kuimba halafu akapokelewa na kundi la wafuasi wake kwa kuimba vipokezi vilivyomo katika wimbo huo. Sherehe za tohara huchukua siku tatu. Siku ya kwanza ni ya kufungua rasmi sherehe za tohara, ya pili ni ya kutembelea jamaa na ya tatu ni ya kutekeleza kitendo chenyewe cha kutahiri vijana. Hii inamaanisha kwamba mhusika mkuu hawezi kuimba mfululizo kwa siku hizo tatu kwa sababu ya kuchoka. Kwa hiyo, ili kumfanya apumzike, viongozi wa kundi la wafuasi wake mara nyingi huanzisha nyimbo nyingine ambazo ni tofauti kabisa na wimbo maalum wa mhusika mkuu. Hata hivyo, utafiti huu umezingatia nyimbo za tohara.

Usanii bora wa wimbo humwongezea sifa mhusika mkuu na kuifanya idadi ya wafuasi wake iongezeke. Vijana mbalimbali hujisifu kwa ustadi wao wa kuimba. Vijana hao pia hujisifu kwa kuwa na idadi kubwa ya wafuasi. Mhusika mkuu akiimba vizuri, yeye huandamwa na watu wengi mkiwemo wanaume na wanawake, wakongwe, vijana pamoja na watoto wadogo kwa sababu wanataka kuendelea kumsikiliza mwimbaji huyo. Hawa wote hushiriki katika sherehe ya tohara kwa kuimba vipokezi vilivyomo katika wimbo wa mhusika mkuu pamoja na kucheza. Utekelezaji wa nyimbo hizi huvuruga mambo mengi na kukwamisha shughuli nyingi katika Wilaya ya Bududa. Hata hivyo, umuhimu wa nyimbo hizi haujajulikana dhahiri, jambo ambalo limechukuliwa kuwa msingi wa kazi hii ya utafiti.

Kwa mujibu wa Kingsriter (1997), muziki wenye asili ya Kiafrika na kucheza ngoma ni mambo ambayo yamedumishwa kwa mdomo kama chombo muhimu na ni tofauti kabisa na muziki wenye tamaduni za Waarabu wa Afrika ya kaskazini na walowezi wa magharibi mwa Afrika ya kusini. Kwa hivyo, muziki wa Kiafrika pamoja na kucheza ngoma ni mambo ambayo yanatekelezwa sana na jamii zinazopatikana kusini mwa jangwa la Sahara. Kulingana na mtaalamu huyu, tamaduni zote zinazopatikana kusini mwa jangwa la Sahara husisitiza uimbaji kwa sababu nyimbo hutumika kama njia muhimu ya mawasiliano. Kwa kuwa lugha nyingi za Kiafrika ni lugha zenye toni ambamo kiwango cha kupanda na kushuka kwa sauti huleta maana, lahani na mahadhi ya nyimbo kwa jumla, hufuata lahani na mahadhi ya matini katika wimbo. Kwa kawaida lahani huandaliwa kwa kipimo cha sauti nne, tano, sita au saba. Katika uimbaji wa pamoja, baadhi ya jamii zimezoea kuimba katika sauti moja au kwa sauti kadhaa, huku jamii zingine zikiimba katika vipande viwili au vitatu. Kwa jumla nyimbo huchukua mtindo wa kuita na kuitikia.

Kwa mujibu wa Kingsriter (1997), tamaduni za Kiafrika zinasisitiza sana uchezaji wa ngoma kwa sababu utendaji unachukuliwa kuwa njia muhimu ya mawasiliano. Kwa sababu hii, uchezaji wa ngoma huhusisha uvaaji wa barakoa, maleba, kujipaka mwili unga wa nafaka na alama zingine zinazoonekana. Katika utendaji, mchezaji anaweza kuchezesha sehemu ya juu ya mwili, pingiti au miguu. Uchezaji pia unaweza kuhusisha sehemu mbalimbali za mwili kwa wakati mmoja na kufanya utendaji kuwa mgumu. Uchezaji wa ngoma unaweza kutekelezwa hadharani bila kuzingatia idadi ya hadhira au unaweza kuhusisha idadi maalum.

Kwa mujibu wa Vendler (1980), tambiko ni kundi la matendo yanayotendeka kwa sababu ya thamani inayowakilishwa ambayo inaweza kuhusu dini au tamaduni za jamii. Tambiko laweza kutekelezwa katika kipindi maalum au katika sherehe maalum au kama jamii inavyopenda. Tambiko laweza kufanyiwa na mtu maalum, kundi la watu au jamii 
nzima katika maeneo maalum yaliyotengwa kwa shughuli hiyo mbele ya watu fulani. Maeneo hayo yanaweza kuwa ya hadhara au ya kisiri. Tambiko laweza kufungamana na sehemu fulani ya watu katika jamii.

Kwa mujibu wa Beier (1967), Waluo ni kabila la watu wanaotekeleza miviga mbalimbali na wanaopenda sana kuimba nyimbo za kiutamaduni. Wataalamu hawa wametaja nyimbo nane za kimapenzi za "Oigo". Nyimbo hizi zilikuwa zikiimbwa na wasichana waliobalehe walipowatembelea wavulana wanaotarajiwa kuwaoa. Wavulana nao wangekaa pale wakisubiri kwa hamu hadi wanapozisikia sauti za wasichana. Baada ya kusikia sauti hizo, mmoja wao angeruka kwa furaha na kutangaza kwamba wasichana wanakuja. Baada ya kuwasili kwao, wasichana wangesimama pale nje huku wakiendelea kuimba nyimbo zao mpaka bikizee wa hapo anapowakabidhi zawadi kama vile pete, mikufu, vipuli na kadhalika.

Kichamu na Odaga (1982) wameshughulikia nyimbo na dansi. Wanasema nyimbo na dansi ni mambo ya kawaida katika jamii za Kiafrika. Kwa mujibu wa wataalamu hawa, karibu kila kazi ya msaragambo huhusishwa na nyimbo pamoja na dansi. Wanasema kuna nyimbo kama vile nyimbo za kazi, nyimbo za vita, nyimbo za matambiko kama zile za tohara, nyimbo za ndoa na nyimbo za matanga. Kulingana na wataalamu hawa nyimbo na dansi ni mambo ambayo yanatawala maisha na mawazo ya watu wa Kiafrika. Kwa kutumia nyimbo watu hufunzwa, hucheka, huomboleza, huliwazana, hutiana moyo na wakati mwingine hupitisha ujumbe wenye siri.

Kwa mujibu wa Chesaina (1991), utamaduni wa Wakalenjini hutawaliwa na nyimbo huku nyimbo mpya zikiwa zikibuniwa kila mara. Nyimbo hizo huainishwa kama: nyimbo za watoto, nyimbo za tohara, nyimbo za ndoa, nyimbo za kejeli, nyimbo za kisiasa na nyimbo za kidini. Mtaalamu huyu anasema nyimbo za Wakalenjini ni sehemu mojawapo ya maisha ya watu. Anaendelea kusema kwamba nyimbo hizo hudhihirisha mitazamo ya watu dhidi ya ulimwengu na kuendeleza utamaduni wao kwa kuhifadhi hisia na mambo ya kijamii. Anasema kwamba kama ilivyo na usimulizi, nyimbo za Wakalenjini hutumika katika ufundishaji ili kustawisha mambo muhimu ya kijamii miongoni mwa vijana na watu wakubwa.

Senkoro (1987) amejadili mifumo mbalimbali ya jamii na kudhihirisha jinsi fasihi ilivyoanzishwa. Senkoro anasema baada ya kuzuka kwa lugha, fasihi ya mwanzo ilifuatia. Anasema kwamba fasihi hii haikutoka kwa Mungu wala haikutokana na hisi tu. Anasema fasihi hii ilitokana na kazi alizozifanya mtu katika kupambana na mazingira yake. Polepole, alianza kutunga maneno na sauti zilizofuatia mapigo ya zana zake za kazi. Baada ya muda, mtu alitumia sauti hizo za mapigo ya zana za kazi kujiimbia nyimbo wakati alipokuwa akifanya kazi. Kwa mujibu wa Senkoro, kuanzia hapo wimbo wa kazi ukawa ndiyo fasihi ya kwanza.

Kwa mujibu wa Njogu na wenzake (2006), kuna ushairi uliotungwa katika miktadha maalum barani Afrika na ushairi wa kimapokezi ulioghaniwa na kuimbwa katika shughuli nyingi za kijamii. Miongoni mwa Wamandika wa Afrika magharibi, Djeli au Griot waliimba na kutukuza uongozi na kuhifadhi katika nyimbo zao matukio muhimu ya kihistoria. Wao pia wanasema, katika sherehe za kijamii kama kwenye ibada, ushairi wa tohara uliweza kutokeza kama hifadhi ya matukio na chombo cha kutuliza nguvu kali zinazoizingira jamii. Wanasema kwamba umahiri wa kutunga nyimbo haukutokana na urithi au mapokezi bali kwa juhudi zenye kutoza jasho.

Nkwera (2004), ameshughulikia sana maana ya wimbo. Anasema kuna bahari kubwa mno ya nyimbo katika fasihi. Baadhi yazo zimeandikwa lakini idadi kubwa zaidi ipo katika fasihi simulizi. Mtaalamu huyu anasema nyimbo huimbwa kwa nafasi na madhumuni mbalimbali. Miongoni mwa nafasi na madhumuni, anataja kumsifu Mola, mashindano ya kujifurahisha na kutumbuiza hadhira, kuitukuza Nchi na mashujaa wake, kushangilia na kukuza mapenzi, kusherehekea na kutukuza kumbukumbu za matukio muhimu mbalimbali maishani na nyimbo za kuhadithia.

\section{MBINU ZA UTAFITI}

Katika utafiti huu, mtafiti alitumia mbinu ya uchunguzi shiriki. Kwa kutumia mbinu hii, mtafiti alizuru nyanjani na kushuhudia utaratibu wa utekelezaji wa matukio ya tohara na kurekodi 
nyimbo zilizokuwa zikiimbwa. Mtafiti alifuatilia matukio hatua kwa hatua mpaka mwisho. Alikuwa na kinasa sauti ambacho alikitumia kunasa nyimbo ambazo zilikuwa zikiimbwa. Baada ya kukusanya nyimbo hizo, mtafiti aliziandika kwenye karatasi halafu akazichanganua.

\section{MAUDHUI MBALIMBALI KATIKA NYIMBO ZA TOHARA ZA WAMASAABA}

Nyimbo za tohara huwa na maudhui. Nyimbo tofauti huwa na maudhui tofautitofauti. Kutofautiana kwa maudhui hutokana na matakwa ya wanasanaa wenyewe ambao ni wahusika wakuu. Hii ni kwa sababu malengo ya wahusika wakuu hutofautiana. Kwa mfano, mhusika mkuu anaweza kuwa na lengo la kutoa shukrani, kuomba kitu, kulalamika, kujisifu, kujihamasisha, kutangaza jambo, kukaribisha na kadhalika. Kwa hiyo, kuna nyimbo zenye maudhui ya kushukuru, kutangaza mambo fulani kama vile kuwasili kwa wageni au mhusika mkuu kutaka kuondoka, nyimbo zenye maudhui ya huzuni, nyimbo zenye maudhui ya lawama, nyimbo za makaribisho, nyimbo zenye maudhui ya kuapa na aina zingine nyingi. Tazama mifano ya nyimbo:

\section{Nyimbo za Makaribisho}

Bakyeni Bolaa: Wageni wanafika

\author{
Lugha asili: Kimasaaba \\ Bakyeni, bolaa \\ Bakyeni, bolaa \\ Baba wankye, bakyeni bolaa \\ Bakyeni, bola \\ Mayi wankye, bakyeni bolaa \\ Bakyeni, bola \\ Kumutambo kwela, bakyeni bolaa \\ Bakyeni, bola \\ Businde bubi, bakyeni bolaa \\ Bakyeni, bola \\ Banu bantsakha, businde bubii \\ Businde, bubii \\ Shalelo, mayii \\ Shalelo, babaa \\ Shalelo, balebee \\ Shalelo, lulwankye \\ Shalelo, ang'ale
}

Wimbo huu uliimbwa na mhusika mkuu kwa kukaribisha wageni mbalimbali. Katika wimbo huu, mhusika mkuu anawajulisha wazazi wake kwamba wageni wameanza kuwasili. Na sababu ya kuwasili kwao ni yeye kuwa umusinde yaani mtu

\author{
Tafsiri \\ Wageni wanafika \\ Wageni wanafika \\ Baba yangu wageni wanafika \\ Wageni wanafika \\ Mama yangu wageni wanafika \\ Wageni wanafika \\ Huzuni yaniua wageni wanafika \\ Wageni wanafika \\ Govi ni baya wageni wanafika \\ Wageni wanafika \\ Watu wananicheka govi ni baya \\ Govi ni baya \\ Leo mama \\ Leo baba \\ Leo jamaa \\ Leo ni siku yangu \\ Leo atanikata
}

ambaye hajatahiriwa. Kisha anaendelea kuwahakikishia kwamba hiyo ni siku yake ya kutahiriwa na lazima atafanya hivyo. Yeye anasema kwamba ana huzuni nyingi kwa kuwaona wageni wakiwasili kwa ajili yake.

\section{Nyimbo za Kuapa}

Ufuatao ni mfano wa nyimbo za kuapa: Bateso Banguta

\section{Lugha asili: Kimasaaba}

Bateso banguta, Bacholi banguta

Umwana omusinde, ndaila ne shalelo o!

Bakhana banguta, ilala ese banguta

Umwana omusinde, ndaila ne shalelo o!

\section{Tafsiri}

Wateso wakanisengenya, Wacholi wakanisengenya

Mwana ana govi, lakini naapa leo...

Wasichana wakanisengenya, kweli mimi wakanisengenya

Mwana ana govi lakini naapa leo... 
Basomi banguta, ilala ese banguta Umwana omusinde, ndaila ne shalelo o! Babukusu banguta, ilala ese banguta Umwana omusinde, ndaila ne shalelo o! Bakikuyu banguta, ilala ese banguta Umwana omusinde, ndaila ne shalelo o! Bakonjo banguta, ilala ese banguta Umwana omusinde, ndaila ne shalelo o!
Wanafunzi wakanisengenya kweli mimi wakanisengenya Mwana ana govi lakini naapa leo...

Wabukusu wakanisengenya kweli mimi wakanisengenya Mwana ana govi lakini naapa leo...

Wakikuyu wakanisengenya kweli mimi wakanisengenya Mwana ana govi lakini naapa leo...

Wakonjo wakanisengenya kweli mimi wakanisengenya Mwana ana govi lakini naapa leo...
Wimbo huu uliimbwa na kijana mmoja ambaye aliapa katika wimbo wake kwamba ni lazima atahiriwe. Katika uimbaji wake, kijana husika anataja makabila mengine yanayotekeleza mambo ya tohara. Miongoni mwa makabila yanayotajwa ni Bukusu, Kikuyu na Wakonjo. Kwa kutaja makabila hayo, mhusika mkuu ameonyesha kwamba wale watu wanaotahiriwa huwacheka wenzao ambao hawajatahiriwa. Kwa ufupi, yeye ameonyesha uhusiano uliopo baina ya makabila yanayotekeleza mambo ya tohara.

\section{Nyimbo Zenye Maudhui ya Huzuni}

Mfano wa nyimbo zenye maudhui ya huzuni ni huu ufuatao: Kumutambo kwamambile

\author{
Lugha asili: Kimasaaba \\ Kumutambo kwa mambile wasala \\ Ishina shakyela ese nasalikha \\ Natamba khwe isintsya khali sindiwo \\ Shalelo netsubile kane muwe \\ Businde shalelo nekanile \\ Bakhana shalelo kane muwe \\ Shalelo netsubile kane muwe \\ Shalelo netsubile kane antye \\ Kamanga'nyu shalelo kane kawe \\ Busani shalelo kane fune \\ Businde shalelo kane buwe
}

\author{
Tafsiri \\ Huzuni imenishika mimi wazazi \\ Kwa nini nikazaliwa mimi \\ Nilikosa pa kwenda, nisingekuwepo \\ Leo nimeapa kumpa \\ Govi leo nimelikataa \\ Nyie wasichana leo nitampa \\ Leo nimeapa kumpa \\ Leo nimekula kiapo atanila \\ Aibu leo itaisha \\ Leo nitakuwa mwanamume kamili \\ Mambo ya govi leo yataisha
}

Katika wimbo huu, mhusika anawajulisha wazazi wake katika mishororo mitatu ya kwanza kuwa ana huzuni nyingi. Yeye pia analalamika kwa nini akazaliwa na anasema kwamba kama angekuwa na mahali pa kwenda asingekuwepo. Hata hivyo, licha ya kuwa na huzuni nyingi, mhusika anasisitiza kuwa lazima atatekeleza mambo hayo ya kiutamaduni.

\section{Nyimbo za Kuaga}

Ufuatao ni mfano wa Nyimbo za kuaga: Inyanga imbii

\section{Lugha asili: Kimasaaba \\ Bakhotsa musibule inyanga imbii \\ Inyanga imbii \\ Bamayi musibule inyanga imbii \\ Inyanga imbii \\ Bakoko musibule inyanga imbii \\ Ingo banindile inyanga imbii \\ Inyanga imbii \\ Bakyeni banindile inyanga imbii}

\section{Tafsiri}

Wajomba naomba niondoke, saa ni mbaya

Saa ni mbaya

Mama zangu naomba niondoke, saa ni mbaya

Saa ni mbaya

Dada zangu naomba niondoke, saa ni mbaya

Saa ni mbaya

Nyumbani wananisubiri, saa ni mbaya

Saa ni mbaya 
East African Journal of Swahili Studies, Volume 2, Issue 2, 2020

Article DOI: https://doi.org/10.37284/eajss.2.2.194

Inyanga imbii

Bakhotsa munonkyise basiku banindile

Basiku banindile

Basiku banindile bakhotsa bankye

Bakhotsa bankyeee

Mukhandekha tawe ilala babii

Ilala babii
Wageni wananisubiri, saa ni mbaya

Saa ni mbaya

Wajomba mnisindikize, maadui wananisubiri

Maadui wananisubiri

Maadui wananisubiri wajomba wangu

Wajomba wangu

Msiniache kamwe, nimo hatarini
Wimbo huu uliimbwa na kijana husika alipotaka kuondoka kwa jamaa zake wa upande wa kukeni na kurudi kwao kwa ajili ya kutahiriwa. Katika wimbo huu mhusika mkuu alisisitiza kwamba saa ni mbaya akimaanisha kuwa wakati wa kurudi nyumbani ulikuwa umefika. Katika wimbo huu pia mhusika mkuu aliwaomba wajomba wamsindikize. Na kama ilivyo kawaida, mhusika mkuu anapotoka upande wa kukeni, yeye anatarajiwa kusindikizwa na jamaa wengi wa upande huo. Idadi kubwa ya jamaa wa upande wa kukeni huwa ni ishara ya mapenzi kwa mpwa wao.

\section{Nyimbo za Kuhamasisha Jamaa}

Ufuatao ni mfano wa Nyimbo za Kutia Jamaa Moyo: Ukhatelema nayola

\section{Lugha asili: Kimasaaba \\ Ukhatelema mayi ilala nayola \\ Ilala nayola \\ Ukhatelema mayi ilala natsowa \\ Ilala natsowa \\ Ukhatelema baba ilala nayola \\ Ilala nayola \\ Ukhatelema baba ilala natsowa \\ Ilala natsowa \\ Bakoko bankye ilala natsowa \\ Basenkye bankye ilala natsowa \\ Basale bankye ilala natsowa \\ Mukhatelema tawe ilala natsowa \\ Mwitse mwibonele ilala natsowa}

\author{
Tafsiri \\ Usitetemeke mama yangu kweli nimetimia \\ Kweli nimetimia \\ Usitetemeke mama yangu kweli nimekua \\ Kweli nimekua \\ Usitetemeke baba yangu kweli nimetimia \\ Kweli nimetimia \\ Usitetemeke baba yangu kweli nimekua \\ Kweli nimekua \\ Dada zangu kweli nimekua \\ Shangazi zangu kweli nimekua \\ Rafiki zangu kweli nimekua \\ Msitetemeke kweli nimekua \\ Njooni mjionee kweli nimetimia
}

Wimbo huu uliimbwa na mhusika mkuu katika sherehe nzima ya tohara. Katika wimbo huu, mhusika mkuu alikuwa akiwahimiza jamaa zake mkiwemo wazazi wake, dada zake, shangazi zake na hata rafiki zake kwamba wasitetemeke kwa sababu alikuwa amekua, kasha akawaita waende washuhudie jinsi angesimama imara. Wimbo huu huibua hisia za kisaikolojia na kumtia nguvu za kumwezesha kukikabili kisu cha ngariba bila hofu.

\section{Nyimbo Zinazotangaza Kuwasili kwa Mhusika Mkuu}

\section{Umwana watabula}

Lugha asili: Kimasaaba

Mubolele papa mulu umwana watabula

Nama wa khotsa bona nola

Mubolele mayi mulu umwana watabula

Nama wa khotsa bona nola

Mubolele kuka mulu umwana watabula

Nama wakhotsa bona nola

Mubolele shikuka mulu umwana watabula

\section{Tafsiri}

Mjulishe baba kwamba mtoto anawasili Natoka kwa mjomba tazama ninawasili Mjulishe mama kwamba mtoto anawasili Natoka kwa mjomba tazama ninawasili Mjulishe babu kwamba mtoto anawasili Natoka kwa mjomba tazama ninawasili Wajulishe jamaa kwamba mtoto anawasili 
East African Journal of Swahili Studies, Volume 2, Issue 2, 2020

Article DOI: https://doi.org/10.37284/eajss.2.2.194

\section{Nama wakhotsa bona nola \\ Mubolele bakyeni mulu umwana watabula \\ Nama wa khotsa bona nola}

Wimbo huu uliimbwa na kijana ambaye alikuwa akitoka kwa mjomba wake wa upande wa kukeni. Alipokaribia nyumbani, kijana huyo alibadilisha wimbo ambao alikuwa akiimba na kuanza kuimba
Natoka kwa mjomba tazama ninawasili

Wajulishe wageni kwamba mtoto anawasili

Natoka kwa mjomba tazama ninawasili

wimbo huu. Wimbo huu, ulikuwa na maana kuwa mhusika mkuu anarudi na yuko karibu kufika nyumbani.

\section{Nyimbo za Lawama}

\section{Were umubi}

\section{Lugha asili: Kimasaaba \\ Were umubi matsima elala omubi \\ Shina shakyele ese umusinde wa mumba \\ Kumutambo wama ee ise wama \\ Ilala kwa ntsila ee ilala kwa ntsila \\ Indtushile ena ee nio emone \\ Basiku bankyi ilala bona betsule \\ Kamakyesi konyene matsima lekhe engume \\ Bakuka befe nabo mbo benkyila}

Tafsiri

Mungu ni mbaya kweli ni mbaya

Kwa akaniumba mvulana

Mateso akanipa kweli akanipa

Mateso yaniua tazama yaniua

Nikimbilie wapi ili niepuke janga hili

Maadui ni wengi tazama wamekusanyika

Busara ya pekee ni kuvumilia

Kwa sababu hata babu zetu walitahiriwa
Mhusika mkuu aliimba wimbo huu kwa kuonyesha malalamiko yake. Katika wimbo huu, mhusika mkuu alikuwa akielekeza lawama kwa Mungu akimshutumu kwa kumuumba mvulana. Maana ni kwamba angekuwa msichana asingekabiliwa na mazito haya. Yeye anajuta kwa nini akazaliwa. Anaendelea kusema kwamba maadui wake ni wengi na jambo la kufanya ni kuvumilia.

\section{Nyimbo Zinazoashiria Hatari ya Tohara}

\section{Isawa niyeyi}

Lugha asili: Kimasaaba
Isawa o-o niyeyi
o-o niyeyi
Isawa niyeyi o-o bona yola
o-o bona yola
Isawa esinalaka o-o niyeyi
o-o niyeyi
Sawa likhumi o-o tsenalaka
o-o tsenalaka
Tsintsakho o-o tsawele
o-o tsawele
Umushebi o-o wanindile
o-o wanindile
Mukhankyelewisa tawe o-o bona tsawele
o-o tsawele
Shifo shikyibole o-o shishankye
o-o shishankye

\section{Tafsiri}

Saa ndio hii

Ndio hii

Saa ndio hii tazama imefika

Tazama imefika

Saa niliyoisema ndiyo hii

Ndiyo hii

Saa kumi ndiyo niliyoisema

Ndiyo niliyoisema

Mazaha umekwisha

Umekwisha

Ngariba ananisubiri

Ananisubiri

Msinicheleweshe, tazama saa imekwisha

Imekwisha

Nafasi ya kwanza ni yangu

Kweli ni yangu 
Mhusika mkuu aliimba wimbo huu saa za jioni, vijana walipokuwa wakikusanywa ili wapelekwe mtoni. Kwa mhusika huyo, hii ilionekana kuwa saa ya hatari kabisa na ni saa ambayo alikuwa akitaja tangu shughuli za tohara kuanza kwamba angetahiriwa. Hii ndio sababu akasema saa imefika, saa niliyoisema.

\section{Nyimbo Zinazoonyesha Utaratibu wa Tohara}

\section{Shalelo nole mulukangu}

\section{Lugha asili: Kimasaaba}

Shalelo nole mulukangu wa Mabala

Inga nolile mulukangu kane ityanyi inkyilye

Inga namile mulukangu ise kane niche

Munafutu balebe khubusane

Bamaukyi fesi kane khusindane

Khulekyikha sawa likhumi kane khukyakale

Buli munu aenza afule ukundi

Ne khukyakala khuli khwobusa

Kamakhuwa kali lukhola khatalu

Mhusika mkuu aliimba wimbo huu katika siku ya kwanza. Katika siku hii, vijana husika hukusanyika kwa mzee mmoja ailiyeteuliwa na wanajamii na kupewa miti shamba ambayo pia huaminika kuwa inawapa vijana nguvu za kukabiliana na kisu cha ngariba. Baada ya shughuli hiyo, vijana hao huzuru

\section{Tafsiri}

Leo nitafika uani kwa Mabala

Kule uani miti shamba nitakula

Nikitoka kule uani nitateremka

Kule Nalufutu tutakusanyika

Wanawe Maukyi sote tutabishana

Kuanzia saa kumi tutacheza

Kila mtu anacheza kuwashinda wengine

Lakini kucheza ni kwa bure

Mambo yenyewe yatakuwa katika siku ya tatu

katika eneo moja maalumu na kuanza kuonyeshana jinsi wanavyocheza. Kwa hiyo katika wimbo huu, kwa kiasi fulani, mhusika alijaribu kuonyesha utaratibu ambao ungefuatwa ingawa hakueleza kikamilifu utaratibu huo.

\section{Nyimbo Zinazoonyesha Umaarufu wa Tohara}

\section{Imalu batenda}

\author{
Lugha asili: Kimasaaba \\ Imalu batenda $\times 2$ \\ Yama khale imalu batenda \\ Imalu batenda $x 2$ \\ Ni shalelo imalu batenda \\ Imalu batenda $\times 2$ \\ Ni kyimiakha kyitsa imalu balitenda \\ Imalu balitenda $x 2$ \\ Mwana wa Mukoya imalu batenda \\ Imalu batenda $\times 2$ \\ Shilulu mbo imalu batenda \\ Imalu batenda $\times 2$ \\ Kane enkyibone imalu batenda \\ Imalu batenda $\times 2$
}

\section{Tafsiri}

Tohara wanaiogopa x 2

Kutoka kale tohara wanaiogopa

Tohara wanaiogopa $\mathrm{x} 2$

Hata leo tohara wanaiogopa

Tohara wanaiogopa $\mathrm{x} 2$

Hata miaka ijayo tohara wataiogopa

Tohara wataiogopa x 2

Mwana wa Mukoya tohara wanaiogopa

Tohara wanaiogopa $\mathrm{x} 2$

Kwamba ni hatari, tohara wanaiogopa

Tohara wanaiogopa $\mathrm{x} 2$

Lakini nitaiona, tohara wanaiogopa

Tohara wanaiogopa $\mathrm{x} 2$
Wimbo huu uliimbwa na mhusika kwa kipindi cha siku tatu. Katika wimbo huu, mhusika alikuwa akidhihirisha jinsi tohara ilivyo na umaarufu na jinsi watu wanavyoiogopa. Mhusika anaendelea kwa kusema kuwa ingawa watu wanaiogopa, wao wamekuwa wakitahiriwa. Maana yake ni kwamba licha ya vitisho vya watu kuhusu tohara, lazima atatahiriwa yaani amejitolea atahiriwe na hatabadili nia yake. 


\section{Nyimbo Zenye Historia ya Tohara}

\section{Imalu yama khale}

\section{Lugha asili: Kimasaaba \\ Ibaibuli ibola ilala ibola Ibraimu enkyila ilala naye enkyila Yakhaba Yesu ilala naye enkyila Bakuka befe ilala nabo benkyila Tsikabila tsinyinkyi ilala tsinkyila Oli ubone Basebei nabo benkyila Oli ubone Babukusu nabo benkyila Oli ubone Bakikuyu nabo benkyila Khengume ee khengume}

\author{
Tafsiri \\ Tohara ni ya kale \\ Biblia inasema kweli inasema \\ Ibrahimu alitahiriwa kweli alitahiriwa \\ Hata Yesu kwa hakika alitahiriwa \\ Babu zetu pia walitahiriwa \\ Makabila mengi pia hutahiriwa \\ Ukitazama Wasebei nao hutahiriwa \\ Ukitazama Wabukusu nao hutahiriwa \\ Ukitazama Wakikuyu nao hutahiriwa
}

Wimbo huu uliimbwa na mhusika kwa kipindi cha siku tatu. Katika wimbo huu, mhusika alikuwa akizungumzia historia ya tohara na anasema kwamba tohara ilianza zamani sana na ametaja watu ambao Biblia inasema walitahiriwa. Hali kadhalika, mhusika alitaja makabila Fulani ya Kiafrika ambayo pia yanatekeleza mambo ya tohara. Miongoni mwa makabila hayo ni: Wasebei, Wabukusu na Wakikuyu.

\section{Nyimbo za Tohara Zinazoashiria Matukio}

Nyimbo za tohara huimbwa vijana husika wanapokuwa wakifanya mambo fulani katika utaratibu wa kutekeleza tohara. Kwa mfano, wao huimba nyimbo hizo wanapokwenda mtoni kuchota maji ya kuandalia pombe na pia wanaporudi nyumbani kutoka huko. Wao pia huimba nyimbo hizo wanapokuwa njiani kwenda kutembelea jamaa zao, kwenda mtoni kupakwa tope kabla ya kutahiriwa na wanapofanya mambo mengine mengi katika mchakato wa kutekeleza tohara.

\section{Nyimbo Zinazoimbwa kwenye Tukio la Kutayarisha Pombe}

Sherehe za tohara huanzia kwa utengenezaji wa pombe ya kienyeji ijulikanayo kama buseela. Pombe hiyo huchukua siku tatu kuwa tayari kunywewa. Kwa hiyo, sherehe za tohara pia huchukua siku tatu, kipindi ambacho huchukuliwa na pombe kuwa tayari. Mambo mawili muhimu yanayofanywa katika siku ya kwanza ni kuandaa pombe, mchakato unaojulikana kwa jina maarufu khukoya na pia kuzuru katika maeneo ya kuzindulia sherehe hizo ndogo ndogo.
Katika siku hii ya kwanza, vijana wanaohusika hukusanyika saa nne hivi penye ua ambao umeteuliwa kwa shughuli ya vijana kutahiriwa. Baada ya vijana hao kukusanyika hapo, wao wanatarajiwa kwenda mtoni kuchota maji ambayo ni muhimu sana katika maandalizi ya pombe. Kama ilivyo kiutamaduni, vijana hao hawapaswi kutumia chombo cha aina yo yote kuchota maji hayo. Ni lazima kuna chungu ambacho kimehifadhiwa kwa muda mrefu na ambacho kazi yake ni ile ya vijana kuchotea maji katika kipindi hiki cha tohara. Chungu hiki huhifadhiwa na mzee mmoja anayeaminika katika jamii. Miongoni mwa vijana hao mkiwemo anayeitwa baba na wenzake, basi huyo ndiye anayetarajiwa kubeba chungu hicho na kuwatangulia wenzake kwenda mtoni na ni yeye ndiye anayetarajiwa kutangulia kutahiriwa. Ikiwa vijana wote wanaitana ndugu, basi mmoja wao hujitolea kwa hiari na kubeba chungu ikimaanisha kwamba ni yeye ndiye atakayewatangulia wenzake kutahiriwa. Kwa kawaida, huyo anayejitolea hujiona kama bingwa.

\section{Nyimbo Zinazoimbwa Vijana Wanapokwenda Mtoni}

Wanapokwenda mtoni kuchota maji, vijana husika huimba nyimbo mahususi zinazofungamana na shughuli ya kutayarisha pombe. Vijana hao huimba nyimbo zenye maudhui ya kwenda mtoni, sababu yenyewe ikiwa ni kuchota maji ambayo wanataka kuyatumia katika kutayarisha pombe. Wanapokwenda mtoni, vijana hao hufuata njia rasmi hata zikipitia katika mashamba ya watu. Kiutamaduni, wao hawaruhusiwi kamwe kukwepa njia hizo. Mfano wa nyimbo zinazoimbwa ni 
East African Journal of Swahili Studies, Volume 2, Issue 2, 2020

Article DOI: https://doi.org/10.37284/eajss.2.2.194

lunakhu lwekhwalinda. Wimbo wenyewe unaelezea hivi:

\section{Lugha asili: Kimasaaba \\ Lunakhu lwekhwalinda ooo nilwolu \\ Ilutsi shalelo ee matsima khukhe \\ Khwekha bona ooo bona khwekhwa \\ Shalelo khukoye ee matsima khukoye \\ Mushibanga wa Siibi kametsi kane khukatae \\ Inyungu nasutile balebee ni mumbone \\ Nayola balebee khengoye \\ Natsoa balebee khengoye}

Tafsiri

Siku tuliyoisubiri ndiyo hii

Mtoni leo lazima tuteremke

Sisi tunateremka ona tunateremka

Leo pombe tutengeneze lazima tutengeneze

Kwa Siibi hapo maji tutachota

Nione mimi chungu nimebeba

Nimetimia acha nitengeneze pombe

Nimekua acha nitengeneze pombe
Wimbo huu huimbwa na kijana anayebeba chungu na kumtangulia mwenzake kwenda mtoni kuchota maji ya kuandalia pombe. Wimbo huu una lengo la kuibua hisia za kisaikolojia miongoni mwa vijana hao na kuwafanya waamini kwamba huu ndio wakati wao na kwamba hawawezi kusuburi tena. Katika wimbo huu, kijana husika anasema kwamba siku waliyoisubiri ndiyo hiyo na kwamba mtoni lazima waende. Yeye anasisitiza kwamba pombe lazima waitengeneze. Hii ni kwa sababu, kama nilivyosema katika sehemu ya matayarisho ya kisaikolojia, inaaminika kuwa pombe huridhisha roho za jamaa waliokufa na hivyo kuwapa vijana husika umahiri. Katika wimbo huu, kijana huyo anatangaza kuwa amekua na anataka awe huru atengeneze pombe. Kama ilivyo kawaida, pombe huwa kama mkataba ambao vijana hao hufanyia wanajamii kusisitiza kwamba wamejitolea na kamwe hawatageuka.

\section{Nyimbo Zinazoimbwa Vijana Wanapotoka Mtoni}

Vijana wanaporudi kutoka mtoni, huimba nyimbo maalum zinazoashiria kurudi kwao. Mfano wa nyimbo kama hizi ni ule wa 'khule ingo balebe.' Wimbo wenyewe unasema:

\section{Lugha asili: Kimasaaba \\ Khule ingo balebee khuleyo \\ Indakano shangumu ee khusekho \\ Isawa yolile ee ye khukoya \\ Shalelo ilala ee khukoye \\ Khukoya nikhwo mboo niye endakano \\ Basali balindile ee khakhukyendake \\ Balebe balindile ee khakhukyendake \\ Inyungu mulukangu ee yalindile \\ Basambwa mulukangu nabo balindile}

\author{
Tafsiri \\ Nyumbani turudi jamaa turudi \\ Mkataba tuuweke sahihi \\ Saa ya kuandaa pombe imefika \\ Leo lazima tuandae pombe \\ Uandaaji wa pombe ndio mkataba \\ Wazazi wamesubiri acha tuharakishe \\ Jamaa wamesubiri acha tuharakishe \\ Chungu pale uani kinatusubiri \\ Roho za waliokufa pia zinatusubiri
}

Katika wimbo huu, kijana husika anasema wanarudi nyumbani watie sahihi zao kwenye mkataba. Kijana huyu anapozungumzia mkataba, huwa anamaanisha maandalizi ya pombe. Hii ni kwa sababu katika mshororo wa tatu yeye anasema kwamba saa ya kuandaa pombe imefika. Na anaendelea kusisitiza kuwa siku hiyo lazima waandae pombe. Na katika mshororo wa tano, kijana huyo anasema wazi kuwa kuandaa pombe ndio mkataba. Yeye pia anasema wazazi wao nyumbani wamewasubiri, chungu kimewasubiri na kwamba hata roho za jamaa wao waliokufa zimewasubiri.

Baada ya kurejea kule uani, kijana aliyebeba chungu humimina maji kidogo katika mchanganyiko wa bidhaa za kuandalia pombe ambazo huwa zimetiwa kwenye chungu kingine kidogo sana na kumkabidhi mwenzake. Vijana hao hukabidhiana chungu namna hiyo hadi kijana wa mwisho. Kijana anayekuwa wa mwisho kumimina maji, yeye pia hutarajiwa kuwa wa mwisho kutahiriwa. 


\section{Nyimbo Zinazoimbwa Baada ya Vijana Kutayarisha Pombe}

Baada ya kutayarisha pombe, vijana husika na jamaa hujumuika barani wakiwa wanaimba na kucheza. Kitendo hiki cha kujumuika katika uchezaji hufanya Wamasaaba wahisi kwamba wako nyumbani. Kitendo hiki hushirikisha watu wa matabaka mbali mbali mkiwemo watu wa kawaida

\section{Lugha asili: Kimasaaba \\ Mwana wa massa shalelo nakoya \\ Indakano mung'ali bona yawele \\ Khukhulibanga emawo ilala byawele \\ Abele ninyaa ne shalelo \\ Bilyo khulya balebe kwe kanile \\ Kane enkyimale o - o nio endye \\ Akhali khukyimala eee sikane endye \\ Lukhoola khatalu o-o kane muwe}

na maofisa serikalini kama vile walimu, madaktari, mahakimu na wabunge. Yaani, hiki ni kitendo cha kuwaleta pamoja. Kwa hiyo, baadhi yao husafiri kutoka miji mbali mbali na hata kutoka nchi za nje kuja kuhudhuria sherehe za tohara. Wanapocheza, vijana hao huimba nyimbo zenye maudhui kwamba wameandaa pombe na hivyo mkataba wametia sahihi. Mfano ni wimbo 'mwana wa massa.' Wimbo wenyewe unaelezea:
Wimbo huu uliimbwa baada ya vijana kukamilisha shughuli ya kutengeneza pombe yaani khukoya. Katika wimbo huu mwimbaji anasema kwamba ametia maji, akimaanisha kuwa maandalizi ya pombe yameanza. Yaani ametia maji kwenye bidhaa za kutengenezea pombe. Na kwake yeye utengenezaji wa pombe ni kama mkataba, ndio akasema mkataba kweli nimetia sahihi. Yeye anaendelea kusema kwamba mambo yamekwisha na hawezi kukwepa mambo hayo tena. Mhusika huyu anasema kwamba siku zote alikuwa anacheza lakini vicheko hivyo havipo tena. Kwa kusema hivi mhusika anamaanisha kuwa amejitolea na hatageuka tena. Kusisitiza hilo, mhusika anasema kwamba moyo wake umekataa kula na kuwa ataimaliza kabla ya kufikiria kula. Yeye anaapa katika mshororo wa saba kuwa bila kuimaliza hatakula. Matamshi haya yanaonyesha kujitolea kwa kijana husika.

Saa za kula zinapofika, vijana hao hurejeshwa nyumbani na kupewa chakula. Vijana wa ukoo mmoja wanatarajiwa kulia chakula hicho pamoja. Kitendo hiki kinaonyesha kwamba kuna umoja katika ukoo huo. Baada ya kumaliza kula, vijana hao huvaa rasmi. Wao huvaa njuga zenye uzito

\section{Lugha asili: Kimasaaba}

Shalelo nole mulukangu wa Mabala

Inga nolile mulukangu kane ityanyi inkyilye

Inga namile mulukangu ise kane niche

\section{Tafsiri}

Mtoto wa massa leo nimetia maji

Mkataba kweli nimeweka sahihi

Hakuna kukwepa tena, mambo yamekwisha

Nilikuwa nikifanya mzaha lakini leo..

Tamaa ya kula imepotea

Nitaimaliza ili niweze kula

Bila kumaliza sitakula

Siku ya tatu o-o nitampa

kiasi kwenye mapaja yao na mapambo mengine yanayoning'inia kwenye miili yao.

\section{Nyimbo za Kwenda Kuhudhuria Uzinduzi wa Sherehe za Tohara}

Baada ya vijana hao kumaliza kuvaa mapambo yao, wao hujipanga pale uani na kupakwa unga wa wimbi ambao huwa umechanganywa kwa maji kabla ya kwenda kwenye maeneo maalumu ambako sherehe za tohara huzinduliwa rasmi. Kazi hii hutekelezwa na mzee ambaye ukoo wake umempa wajibu huo. Kama ilivyo kawaida, huyu huwa ni mzee ambaye anaaminika na ukoo wake kuwa yeye si mchawi.

\section{Nyimbo za Vijana Kwenda Kula Dawa za Miti Shamba}

Wanapokuwa njiani kwenda kula dawa za miti shamba, vijana husika huimba nyimbo zinazoonyesha kwamba wanakwenda kwa mzee huyo maarufu kula miti shamba, yaani ityanyi. Mfano ni wimbo shalelo nole mulukangu. Wimbo unaelezea:

\section{Tafsiri}

Leo nitafika uani kwa Mabala

Kule uani miti shamba nitakula

Nikitoka kule uani nitateremka 


\section{Munafutu balebe khubusane \\ Bamaukyi fesi kane khusindane \\ Khulekyikha sawa likhumi kane khukyakale \\ Buli munu aenza afule ukundi \\ Ne khukyakala khuli khwobusa \\ Kamakhuwa kali lukhola khatalu}

Mhusika mkuu aliimba wimbo huu walipokuwa njiani wakienda kula dawa za miti shamba kabla ya kwenda kuhudhuria uzinduzi wa tohara. Hii ni kwa sababu, siku hiyo vijana husika hukusanyika kwa mzee mmoja aliyeteuliwa na wanajamii na kupewa dawa za miti shamba ambazo pia huaminika kuwa zinawapa vijana nguvu za kukabiliana na kisu cha ngariba.

Baada ya shughuli hiyo ya ityanyi, vijana hao huzuru eneo moja maalum na kuanza kuonyeshana jinsi wanavyocheza. Kwa hivyo wimbo huu, kwa kiasi fulani, ulitumiwa na mhusika kuonyesha utaratibu wa uchezaji utakaofuatwa. Katika wimbo huu mhusika huyu anasema leo ni lazima afike kwa Mzee Mabala na huko atakula miti shamba. Anaendelea kusema kwamba akitoka huko, atateremka na katika uwanja wa Nalufutu ataungana na wenzake kutoka koo zingine. Baada ya kuungana, wao watabishana katika uchezaji kuanzia saa kumi. Yeye anasema wazi kuwa katika mashindano hayo, kila mhusika atacheza akiwa na lengo la kuwashinda wenzake. Mhusika huyu anahitimisha katika wimbo wake kuwa kucheza huko ni kwa bure kwa sababu mambo yenyewe yatakuwa siku ya mwisho.

Wanapofika mahala wanapostahili kula ityanyi, vijana husika hujipanga uani kisha Mzee Mabala akawaletea dawa hizo na kuwalisha. Inaaminika kuwa dawa hizo huwaongezea vijana umahiri na ukakamavu na hivyo kuwawezesha kukikabili kisu cha ngariba bila hofu.

Baada ya vijana kula dawa hizo za miti shamba, wao huondoka moja kwa moja kwenda kwenye uwanja wa kuzindulia sherehe hizo za tohara. Misafara ya kwenda uwanjani hujumuisha vilevile nyimbo na uchezaji. Shamrashamra hizo huelekezwa katika njia mahsusi zilizoamuliwa awali na wazee maalum. Uamuzi wa wazee hawa huwa haupingwi hata inapokuwa bayana wakati mwingine kuwa njia walizozichagua
Kule Nalufutu tutakusanyika

Wanawe Maukyi sote tutabishana

Kuanzia saa kumi tutacheza

Kila mtu anacheza kuwashinda wengine

Lakini kucheza ni bure tu

Mambo yenyewe yatakuwa katika siku ya mwisho

zingesababisha uharibifu mkubwa. Kwa mfano, binafsi nilishuhudia rasmi zikipitia mashambani mwa watu; jambo ambalo lilisababisha uharibifu mkubwa wa mimea. Hilo linapotokea, wanajamii wanatarajiwa waone kwamba hayo yanatekelezwa kwa faida pana ya jamii nzima. Katika Wilaya ya Bududa, sherehe hizo za uzinduzi wa tohara hufanywa kwenye maeneo maalum kama vile Nalufutu katika mtaa wa Bukigai na Namasho katika mtaa wa Bulucheke, maeneo ambayo yametengwa kwa shughuli hiyo. Koo zote za Bukigai hukusanyika kule Nalufutu. Na koo zote za Bulucheke hukusanyika kule Namasho. Koo zote za Bududa nazo hukusanyika kule Nashulo. Lakini sherehe hizo hutekelezwa siku tofauti tofauti kulingana na ratiba, yaani kila mtaa una siku yake maalum.

Baada ya vijana kula dawa za miti shamba, wote pamoja na wafuasi wao huzuru kwenye maeneo hayo maalum. Wakiwa hapo, wao huimba na kucheza kama wanavyoweza. Kila mhusika mkuu hujaribu kuimba na kucheza kuwashinda wenzake wote. Wafuasi wao pia hufurahia sherehe hizo kwa kucheza kama wanavyopenda.

\section{Nyimbo Zinazoimbwa Vijana Wanapotembelea Jamaa Zao}

Siku ya pili, vijana husika pamoja na wafuasi wao hutembelea jamaa zao mbali mbali mkiwemo halati, shangazi, marafiki wa wazazi wao, na dada zao. Wakiwa huko, wao huandaliwa vyakula mbali mbali. Kwa mfano, wao huchinjiwa kuku, mbuzi, ng'ombe na kupewa zawadi zingine tofauti tofauti. Kama Shilimi (1978) anavyosema tohara ni mviga unaoleta wanajamii pamoja kwa ajili ya kunywa na kula, pombe Pia huandaliwa. Kijamii, jambo la kutembelea jamaa katika kipindi hiki ni muhimu. Kwa sababu hii ni ishara kuwa kuna umoja, ushirikiano na utegemeano miongoni mwa wanaukoo. Wanapokuwa njiani wakienda, wao huimba na kucheza mpaka wanapofika kule 
wanakokwenda. Siku hiyo ni ya kuburudika tu. Yaani ni siku ya kuimba, kucheza na kula. Vijana ambao hawana jamaa, siku hiyo wao huchezeachezea njiani. Kwa kawaida, vijana

\section{Lugha asili: Kimasaaba \\ Nayola basanii nayola \\ Natsowa balebee natsowa \\ Kane ngubone eee kane ngubone}

Katika wimbo huu, mhusika mkuu anaihakikishia jamii yake kuwa amekua na yuko tayari kukabiliana na kisu cha ngariba bila hofu yo yote. Wimbo huu pia huibua hisia za kisaikolojia na kumtia nguvu za kumwezesha kukabiliana na ngariba. Kwa mfano, katika wimbo huu mhusika mkuu anasema kwamba ametimia, amekua na atakiona, akimaanisha kwamba atakiona kisu cha ngariba.

\section{Lugha asili: Kimasaaba \\ Senkye wankye ise netsile \\ Iwe walankyila baba welakho \\ Bisela bikali mubele mwa kwana \\ Uwabasala enywe umukhana wa Kuremu \\ Umwana wa Kusolo ee Ibukhwaka \\ Kyakala senkye ee shalelo \\ Kyibole wowo ee niyoyu \\ Ilala emalu ee yama khale \\ Kane enkyimale ee kusenkye}

Hapa inabainika kuwa kijana husika aliimba wimbo huu alipowasili kwa shangazi yake. Katika wimbo huu, kijana husika anaanza kwa kumjulisha shangazi yake kuwa amewasili. Yeye anaonyesha uhusiano uliopo baina yao kwa kumwambia shangazi yake kuwa alitangulia baba yake kwamba yeye na shangazi yake wana uhusiano wa damu. Mhusika anaendelea kusema kwamba shangazi yake na baba yake wanapendana na kwamba aliyewazaa ni binti wa Kuremu kutoka Bukhwaka. Kijana huyu anasema kwamba yeye ni kitinda mimba. Lengo kuu la kufanya hivyo ni kumshawishi shangazi ampe zawadi. kuzaliwa. Hapa, kijana husika anajaribu kuonyesha wanapokuwa njiani kwenda kwa jamaa zao, wao huimba nyimbo zenye maudhui tofauti kama ufuatao:
Tafsiri

Nimetimia wanaume nimetimia

Nimekua jamaa zangu nimekua

Nitakiona eee nitakiona
Vijana wanapowasili kwa jamaa zao, wao huimba nyimbo zenye maudhui ya kuwasili. Nyimbo zinazoimbwa hutofautiana kulingana na uhusiano uliopo na mtu ambaye ametembelewa. Ikiwa jamaa aliyetembelewa ni halati, basi mhusika atamtaja hivyo katika uimbaji wake. Yaani, sifa zinazotolewa na mhusika katika wimbo wake hutegemea uhusiano uliopo baina yake na jamaa ambaye ametembelewa. Mfano ni wimbo 'senkye wankye.'

\section{Tafsiri}

Shangazi yangu nimefika

Ulitangulia kuzaliwa baba akafuata

Kwa muda mrefu mlipendana

Aliyewazaa ni bintiye Kuremu

Msichana wa Kusolo kule Bukhwaka

Cheza leo ee Shangazi

Kifungua mimba ee ndio huyu

Kwa kweli tohara ilianza zamani

Nitaimaliza ee Shangazi

\section{Nyimbo Zinazoimbwa Vijana Wanapokwenda Upande wa Kukeni}

Siku ya tatu, kitendo chenyewe cha kutahiri vijana kinatekelezwa. Hii pia ni siku ya vijana husika kutembelea jamaa zao wa upande wa kukeni. Wakati wa kuondoka kwenda upande huo hutegemea umbali uliopo. Wale wanaokwenda mbali huondoka mapema huku wenzao wanaokwenda karibu huondoka kama saa sita hivi. Kabla ya kuondoka, vijana hao hujipanga pale uani wakiwa wamevaa rasmi na kupakwa unga wa wimbi tena. Baada ya shughuli hiyo, vijana husika pamoja na wafuasi wao huondoka na kwenda kule alikozaliwa mama. Kwa kawaida, vijana hao huenda huko kama baba zao walilipa mahari. Bila mahari kulipwa, vijana hawawezi kwenda huko. Hata hivyo, kuna jamaa wengine wa upande wa kukeni ambao wanaweza kumwita mpwa wao na 
kumpa zawadi ingawa baba yake hakulipa mahari. Wanapokuwa njiani wakienda, mhusika huimba nyimbo kama ubeti huu ufuatao:

\section{Lugha asili: Kimasaaba \\ Kumutambo kwa mambile wasala \\ Ishina shakyela ese nasalikha \\ Natamba khwe isintsya khali sindiwo \\ Shalelo netsubile kane muwe \\ Businde shalelo nekanile \\ Bakhana shalelo kane muwe \\ Shalelo netsubile kane muwe \\ Shalelo netsubile kane antye \\ Kamanga'nyu shalelo kane kawe \\ Busani shalelo kane fune \\ Businde shalelo kane buwe}

\section{Tafsiri}

Huzuni imenishika mimi wazazi

Kwa nini nikazaliwa mimi

Nilikosa pa kwenda, nisingekuwepo

Leo nimeapa kumpa

Govi leo nimelikataa

Nyie wasichana leo nitampa

Leo nimeapa kumpa

Leo nimekula kiapo atanila

Aibu leo itaisha

Leo nitakuwa mwanamume kamili

Mambo ya govi leo yataisha
Wimbo huu huimbwa katika siku ya tatu. Katika wimbo huu, mhusika anawajulisha wazazi wake katika mishororo mitatu ya kwanza kuwa ana huzuni nyingi. Yeye pia analalamika kwa nini akazaliwa na anasema kwamba kama angekuwa na mahali pa kwenda asingekuwepo. Hata hivyo, licha ya kuwa na huzuni nyingi, mhusika anasisitiza kuwa lazima atatekeleza mambo hayo ya kiutamaduni.

Wakiwa huko, wao pia huandaliwa vyakula mbali mbali na pombe. Vijana hutembelea jamaa zao wote wa upande huo wa kukeni. Hii ni ishara kuwa kuna umoja katika ukoo wa mama. Katika ziara zao hizo, vijana husika hupewa zawadi mbali mbali. Kama ilivyo kawaida, zawadi wanazopewa hutegemea mahari yaliyotolewa na akina baba. Ikiwa baba wa kijana fulani alitoa mahari ya kuridhisha, yaani pesa nyingi au ng'ombe wanaopendeza, basi mvulana wake pia huzawadiwa vizuri na kwa moyo mkunjufu.

\section{Lugha asili: Kimasaaba \\ Nambakha netsa shinume \\ Nambakha netsa imuwe \\ Nalaba netsa imuwe \\ Nakhula netsa imuwe \\ Nayola netsa imuwe \\ Nafuma netsa imuwe \\ Balebe mutse mubone}

\section{Nyimbo Zinazoimbwa Vijana Wanapokwenda Kutahiriwa}

Baada ya kupakwa tope na kuosha dhakari, vijana huongozwa na wazee wale wale kwenda kwenye eneo ambalo limetengwa kwa shughuli ya kutahiri vijana. Wakati wa kutoka mtoni ni hatari sana kwa vijana husika. Hii ni kwa sababu ya uchungu unaotokana na tohara. Wakati huu vijana huwa katika hali ambayo si ya kawaida. Kwa sababu hiyo, vijana huwa wanaimba nyimbo za kuathiri hisia zao kisaikolojia kwa madhumuni ya kujipa moyo wa kutaka kukabiliana na ngariba. Wafuasi wao pia huimba nyimbo za aina hiyo. Hii ni kwa sababu watu wengi wanaamini kwamba vijana wakifanyiwa vituko hivyo mbali mbali, wao huongezewa nguvu fulani zinazowawezesha kutekeleza tohara bila kuwa na hofu. Hii ndiyo sababu wazazi wengi hawataki vijana wao kwenda hospitalini kutahiriwa. Mfano wa nyimbo zinazoimbwa vijana wanapotoka mtoni ni ule wa 'nambakha netsa.'
Vijana wengi huimba wimbo huu wanapotoka mtoni wakielekea uani kutahiriwa. Inaonekana

\section{Tafsiri}

Mpinzani naja kiniume

Mpinzani naja nimpe

Nimepevuka naja nimpe

Nimepevuka naja nimpe

Nimetimia naja nimpe

Nimejulikana naja kiniume

Jamaa zangu mje muone wimbo huu huwatia vijana mori sana. Katika wimbo huu, mhusika mkuu anaimba kwa sauti ya 
kishujaa akisema kwamba yeye ni mpinzani sasa anakuja, maana yake ni kwamba yeye ni shujaa na anakuja. Anaendelea kusema kwamba yeye amepevuka, ametimiza umri, amejulikana sana na sasa anakuja kumkabili ngariba. Yeye anatamka maneno haya kujipa moyo na kuwaondolea jamaa zake hofu.

\section{HITIMISHO}

Makala hii inaonyesha kuwa jamii ya Wamasaaba ni miongoni mwa jamii nyingi za Kiafrika ambazo zina rundo la fasihi simulizi. Vijana wengi wamebobea katika uwanda huu wa fasihi. Nyimbo hizi ni njia mojawapo ya kuingizia serikali ya Uganda pesa kwa sababu wakati wa utendaji, watalii huwa wakihudhuria. Na hata jamii yenyewe huwa inafaidika kutokana na uuzaji wa vitu vya kienyeji kwa wageni. Kwa hiyo, juhudi zinastahili kufanywa na serikali ya Uganda ili kuendeleza utamaduni huu kwa madhumuni ya kunufaisha zaidi jamii husika. Hali kadhalika, utafiti huu unaonyesha kuwa utamaduni si jambo la kishenzi kama watu wengi wanavyofikiria. Kwa kuzingatia matokeo ya utafiti huu, inabainika wazi kuwa utamaduni ni msingi wa maisha ya binadamu. Ni kweli kwamba tamaduni zetu si duni kama watu wengi wanavyokisia. Ni jambo la kusikitisha kuona kwamba ni tamaduni chache sana za Kiafrika ambazo zimetafitiwa kufikia sasa. Kwa hiyo, wanafasihi wanastahili kuangazia jambo hili kwa kina.

Ikiwezekana, CHAMA cha kuendeleza utafiti kuhusu tamaduni za Kiafrika kiundwe. CHAMA kama hicho kikiundwa, bila shaka kitaangazia tamaduni mbalimbali za kiafrika na kuhakikisha kwamba tamaduni hizo zinashughulikiwa vilivyo. Ili jambo hili liweze kufanyika, kuna mahitaji ya nchi zetu za kiafrika kuanzisha mfuko wa pesa za kufadhili CHAMA hicho. Hii inamaanisha kuwa muungano wa nchi za afrika (OAU) unastahili kuchukua hatua. Bila kufanya hivyo, tamaduni nyingi za kiafrika zitaangamia na sote tutakuwa tukienzi tamaduni za ughaibuni. Hii ni kwa sababu waafrika wengi wanachukulia tamaduni zao asili kuwa duni.

\section{MAREJELEO}

Beier, U. (1967). Introduction to African literature: an anthology of critical writing from Black Orpheus. Evanston IL: Northwestern University Press.

Chesaina, C. (1991). Oral Literature Of the Kalenjin. Nairobi: East Africa Educational Publishers Ltd.

Kichamu, S. \& Odaga, A. (1982). Oral Literature, A School Certificate Course. Nairobi: Heinemann Educational Books (E.A) Ltd.

Mlacha, S. A. K. (1995). Lugha, Utamaduni na Fasihi Simulizi. Taasisi ya uchunguzi wa Kiswahili, Chuo Kikuu cha Dares Salaam.

Nangoli, M. (2001). No more lies about Africa: here's the truth from an African. Brooklyn, NY: A $\&$ B Publishers Group.

Njogu, K; Momanyi, C. \& Mathooko, M. (2006). Fasihi Simulizi ya Kiswahili. Nairobi: Twaweza Communications.

Nketia, J. K. (1955). Funeral dirges of the Akan people (Vol. 2). Negro Universities Press.

Nkwera, F.F.V. (1989). Tamrini za Fasihi Simulizi. Dar es Salaam: Kitabu Commercial Printing Co. Ltd.

Senkoro, F. E. M. K. (1987). Fasihi na Jamii. Dar es Salaam: Press and Publicity Centre.

Vendler, Z. (1980). Telling the facts. In Speech act theory and pragmatics (pp. 273-290). Springer, Dordrecht. 